\title{
Topology Preservation and Tricky Patterns in Gray-Tone Images
}

\author{
Carlo Arcelli and Luca Serino \\ Istituto di Cibernetica "E. Caianiello", CNR \\ 80078 Pozzuoli, Napoli, Italy \\ \{c.arcelli,1.serino\}@cib.na.cnr.it
}

\begin{abstract}
A gray-tone image including perceptually meaningful elongated regions can be represented by a set of line patterns, the skeleton, consisting of pixels having different gray-values and mostly placed along the central positions of the regions themselves. We discuss a skeletonization algorithm, computed over the Distance Transform of the image and employing topology preserving operations. Differently from the binary case, where the use of the connectivity test is generally sufficient to create a one-pixel-thick skeleton, we consider also a suitable labeling of the pixel neighborhood. In this way, we are able to deal with some of the tricky patterns in the gray-tone image that can be regarded as irreducible.
\end{abstract}

\section{Introduction}

In gray-tone digital images, regions with locally higher gray-value can be understood in certain problem domains as the ones carrying the most relevant information. This is the case when an image includes perceptually significant elongated subsets, generally constituted by pixels characterized by different gray-values. This meaningful information can conveniently be represented in terms of a set of line patterns, called skeleton hereafter, generally consisting of pixels having different gray-values and mostly placed along the central positions of the regions themselves.

If a gray-tone digital image is regarded as a mountainous relief, the gray-value of a pixel being its height, the identification of the skeleton can be related to the detection of topographic features such as ridges, peaks and saddles. For instance, the skeleton could be found by considering the image as a continuous surface, and by using the first and second partial derivatives of this surface to identify the skeletal pixels [1]. Alternatively, one could consider the gray-tone image as an ordered set of binary images each one obtained, by suitably thresholding, as a cross-section of the graytone image [2]. Skeletonization is then accomplished by repeatedly lowering the gray-value of certain pixels until gray-values which characterize regional minima are eventually assigned to them [3]. Generally, a lowering operation should not modify the topology of the gray-tone image, in the sense that any cross-section binary image should preserve its topology [4]. 
In this paper, we describe a skeletonization algorithm driven by the Distance Transform of the gray-tone image. Particularly, to find a skeleton placed along the proper medial positions, we exploit the structural information characterizing the Distance Transform of a single-valued region and take into account the dominance relations among the regions constituting the gray-tone image.

We regard the image as piecewise constant [5] and for each region with constant gray-value we compute the Distance Transform. The latter is of the constrained type [6] whenever there exist adjacent regions with higher gray-value. Computation of the Distance Transform is accomplished according to the $(3,4)$-weighted distance [7], by ordered propagation over regions with increasing gray-values. The pixels in each region receive a distance label related to their geodesic distance from a reference set constituted by the pixels with lower gray-values adjacent to the region. Then, the pixels are examined in a suitable order, and the ones that are end points or non simple points are taken as elements of the skeleton.

Due to the possible complexity of the morphology of a gray-tone image, topology preserving reduction operations are not always sufficient to create a one-pixel-thick skeleton. In this respect, we consider a suitable labeling of the pixel neighborhood, which allows us to deal with some tricky patterns in the gray-tone image that can be regarded as irreducible.

We note that both a preprocessing phase and a postprocessing phase should be included in any skeletonization algorithm applied to real world images. The role of the preprocessing is to remove narrow peaks and pits as well as to fill in valleys and flatten plateaux. In turn, the postprocessing phase is required to remove skeleton branches which do not constitute significant separations (watersheds) between adjacent basins, and to prune branches which do not denote significant promontories. We are not specifically interested in these phases and will only mention some features of the preprocessing phase we take into account.

\section{Preliminaries}

Let $G$ be a gray-tone digital image. Pixels in $G$ are assigned one out of a finite number of increasing integer values $g_{k}, k=0,1, \ldots, \mathrm{N}$, which indicates for any pixel $p$ the gray-value or status $g(p)$ of the pixel itself. Letters will be used to denote both pixels and their gray-values. We assume that $G$ is bordered by a frame of pixels with grayvalue greater than $g_{N}$.

The neighbors of $p$ are its 8 -adjacent pixels. They constitute the neighborhood $N(p)$ of $p$ and are denoted by $n_{l}, n_{2}, \ldots, n_{8}$, where the subindexes increase clockwise from the pixel $n_{1}$ placed to the left of $p$. The neighbors $n_{i}, i$ odd, are called direct neighbors (d-neighbors). The neighbors $n_{i}, i$ even, are called indirect neighbors (ineighbors). We denote by max and $\min$, respectively, the maximal gray-value and the minimal gray-value of the $n_{i}$ having gray-values less than $g(p)$.

If $p>n_{i}$, for at least one d-neighbor, $p$ is termed lower border point. If $p<n_{i}$, for at least one d-neighbor, $p$ is termed upper border point. If $p$ has only one neighbor or just two consecutive neighbors with gray-value equal to its own gray-value and all the remaining neighbors have smaller gray-value, $p$ is termed end point. 
A gray-tone image can be regarded as a mosaic, generally made of very irregular pieces (or regions), different in gray-value, shape and size. The regions do not overlap each other and are maximal 4-connected sets of pixels with a same gray-value. Two regions with different gray-value are called adjacent if they are 4-adjacent. A bottom region (shortly, a bottom) is a region with all its adjacent regions having higher gray-values. Any bottom is a regional minimum of $G$. A top region (shortly, a top) is a region with all its adjacent regions having lower gray-values.

When $G$ has to be processed by using topology preserving operations, it is necessary to identify for each of its pixels which are the foreground and the background and which kind of connectedness holds for each of them. Thus, rather than as a mosaic, it is convenient to understand $G$ as a stack of binary images, by following an approach dating back to the studies on threshold logic [8] and widely used in the literature. More in detail, for any gray-value $g_{k}$, with $k$ different from 0 , the gray-tone image is regarded as a binary one, say $B_{k}$, where the set of pixels having gray-values not less than $g_{k}$ constitutes the current foreground and the set of pixels having grayvalues less than $g_{k}$ constitutes the current background. Thus, according to the chosen threshold values, there are $\mathrm{N}$ binary images in correspondence with $G$. In $B_{k}$, the 8connectedness should be understood to hold for the foreground, the 4-connectedness for the background.

When applying operations which change the status of some pixels, we say that the topology of the gray-tone image does not change if none of the $\mathrm{N}$ binary images $B_{k}$, found in correspondence with the various thresholds, has its topology changed.

In a binary image, the assignement of a pixel $p$ to a component, different from the one it currently belongs to, changes the topology of the image whenever it causes a modification in the number of components of the foreground or of the background. In this respect, topology is preserved if ones removes from the foreground (i.e., assigns to the background) only the pixels, termed simple points, which satisfy certain neighborhood conditions. For instance, simple points are those $p$ for which the 8connectivity number $C(p)$ is equal to one [9].

$$
C(p)=\Sigma_{\mathrm{i} \text { odd }}\left(\left(1-n_{i}\right)-\left(1-n_{i}\right)\left(1-n_{i}+1\right)\left(1-n_{i}+2\right)\right)
$$

When considering a pixel $p$ in a gray-tone image, we should refer to the binary image $B_{k}$, where the threshold $t=g_{k}$ corresponds to $g(p)$. By regarding $n_{i}$ as a Boolean variable equal to 1 if $n_{i} \geq p$, and equal to 0 otherwise, the 8-connectivity number $C(p)$ for a lower border point $p$ turns out to be equal to the number in $N(p)$ of 8 components of pixels with gray-value not less than $g(p)$. We say that $p$ is a simple point of $G$ if it is a simple point of $B_{k}$, namely if $C(p)=1$ in $B_{k}$.

End points and pixels that are not simple points are called feature points.

A reduction operator is an operator which replaces the gray-value of a pixel by the gray-value of one of its neighbors having smaller gray-value. A reduction operator is topology preserving in $G$ whenever it is applied only to simple points and lowers to $\max$ the gray-value of each of them $[4,10]$.

Let $X$ and $X^{c}$ respectively denote a region of $G$ and its complement, and suppose that $R$ is a subset of $X^{c}$ adjacent to the whole border of $X$. The Distance Transform of $X$ with respect to the reference set $R$ is the multi-valued set $D T(X, R)$, which differs from $X$ in having each pixel labelled with its distance from $R$, computed according a 
chosen distance function. If $R$ is not adjacent to the whole border of $X$, the transform is called the constrained Distance Transform of $X$.

\section{Skeletonization}

In this section, we outline a skeletonization algorithm driven by the Distance Transform of the gray-tone image and based on the use of topology preserving reduction operations. Moreover, we briefly discuss the preprocessing phase which is often crucial to obtain meaningful results. In summary, the main steps leading to the creation of the skeleton are the following:

1. Preprocessing;

2. Distance transformation;

3 . End point detection;

4. Lowering of simple points present in successively adjacent regions with increasing gray-value;

5. Postprocessing.

\subsection{Preprocessing}

The scope is to create a image having only a number of significant (i.e., deep enough) bottoms and a number of tops not too crenelated. Bottoms and tops of the input image are taken as seeds and, in correspondence with them, we construct multi-level $(\varepsilon, \delta)$-components [11]. Each component is then identified by the gray-value of the corresponding seed. A multi-level $(\varepsilon, \delta)$-component is a region where the difference in gray-value between two d-neighbors never exceeds the adjacency parameter $\delta$, and the maximum difference in the gray-values of its pixels does not exceed the range parameter $\varepsilon$. Moreover, any component satisfies a maximal property, i.e., no valid component can be merged with an adjacent valid component to form a larger valid component. It has been pointed out that the values of $\varepsilon$ and $\delta$ can conveniently be adjusted for different types of images or different levels of analysis. In this paper, we refer to input images characterized by 256 gray-levels and relate $\varepsilon$ to the greatest difference in gray-value $\Delta$ between adjacent d-neighbors. Particularly, we set $\varepsilon=\Delta-1$. The rationale for this choice is to ensure a distinction between the foreground and the background in a binary image. As for $\delta$, we select the value $\lfloor\Delta / 2\rfloor$.

Tops and bottoms are identified and then grown into $(\varepsilon, \delta)$-components, which are created by iteratively aggregating to each seed the $\delta$-adjacent regions. The output of this phase is a modified image $G^{*}$ where the obtained $(\varepsilon, \delta)$-components take the place of the corresponding regions in the initial image.

\subsection{Distance Transformation}

We regard the gray-tone image $G^{*}$ as the union of a number of single-valued regions, and compute the Distance Transform of every region with respect to a reference set constituted by the regions with lower gray-values and adjacent to the region. 
The Distance Transform of the gray-tone image is the union of the (constrained and unconstrained) Distance Transforms of the regions constituting the image.

A region is classified according to three typologies, depending on the gray-values of the adjacent regions:

type 1. All the adjacent regions have smaller gray-values;

type 2. Only some of the adjacent regions have smaller gray-values;

type 3. All the adjacent regions have greater gray-value.

It is straightforward to observe that for the regions of type 1 , the Distance Transform is unconstrained since there are no adjacent regions with higher gray-values. These regions are characterized by locally higher intensities and will certainly include a skeleton branch.

For any region of type 2, the Distance Transform is constrained and its computation leads to a set of propagating wave fronts (each wave front being a connected set of pixels with a same distance label) which interact with each other whenever the region protrudes over adjacent regions with smaller gray-value.

A region of type 2 is perceptually dominated by the adjacent regions with higher gray-value, and the strength of this dominance is as greater as less protruding is the region itself. Let $X$ and $Y$ denote two regions of $F$, with $g(X)<g(Y)$, and let $L_{X}$ and $L_{X Y}$ be the lengths of the border of $X$ and of the part of the border of $X$ adjacent to $Y$, respectively. We say that $X$ is not strongly dominated by $Y$ if $\left(L_{X}-L_{X Y}\right)>3 L_{X Y}$. Our procedure detects significant skeleton subsets only in correspondence with regions that are not strongly dominated.

Finally, for the regions of type 3 the Distance Transform cannot be computed since all border pixels are adjacent to regions with higher gray-values and the reference set is empty.

The computation is accomplished on the array where the preprocessed gray-tone image is stored, and is performed according to the $(3,4)$-weighted distance, by ordered propagation over regions with ascending gray-values. Queues, i. e., first-infirst-out data structures, are used to this purpose. The lower border points of the image are traced and stored in a priority queue constituted by a set of queues, which have different priority levels. The use of the priority queue allows one both to minimize pixel access and to process efficiently pixels in increasing order of gray-value. The priority levels correspond to the increasing gray-values in the image, higher priority corresponding to lower gray-value. The queue at priority level $g_{k}$ contains only the pixels with gray-value $g_{k}$ currently under examination. As a result, the pixels in each region receive a distance label related to their geodesic $(3,4)$-weighted distance from a reference set constituted by the pixels with lower gray-values and adjacent to the region. If $X_{k}$ denotes the region(s) with gray-value $g_{k}$, the distance labels in $X_{k}$ turn out to be smaller (greater) than those in $X_{k}+1\left(X_{k}-1\right)$.

\subsection{End Point Detection and Pixel Lowering}

We refer to the Distance Transform of $G^{*}$ and follow the classical scheme which is concerned first with the detection of the end points present in every region of the 
image (they are marked as feature points), and successively, starting from the lower border points of every region, with the iterated lowering of more and more internal pixels.

The definition of end point in the Distance Transform is given in terms of distance label. Specifically, a pixel $p$ is defined end point if it has only one neighbor or just two consecutive neighbors with distance label equal to its own distance label and all the remaining neighbors have smaller distance label. Moreover, to cope with the discrete nature of the digital plane we mark also two-pixel-thick end point configurations such as the set of $p$ 's in Fig.1.

$\begin{array}{llll}c & c & c & c \\ c & p & p & c \\ c & p & p & c \\ c & a & a & c\end{array}$

Fig. 1. The 4-tuple of $p$ 's is a two-pixel-thick end point configuration. Pixels $c$ 's have distancelabels less than $p$, pixels $a$ 's have distance-labels not less than $p$.

End point detection is performed on the Distance Transform during one scan of the image.

As for pixel lowering, the same data structure adopted for the computation of the Distance Transform is used to access the pixels of each region, according to the increasing value of their distance from the lower border points. For every set of pixels with the same distance label, the connectivity test is performed repeatedly until only pixels that are end points or not simple points are left.

When computing the 8-connectivity number on a pixel $p$, we refer to its binary neighborhood where a neighbor is regarded equal to 1 if it is a feature point or has distance label not less than the distance label of $p$, and equal to 0 otherwise.

The pixels that are simple points are lowered, while the remaining ones are marked as feature points. Once a region has been completely examined, the process is repeated on the successive regions with greater gray-value, until the image is exhausted.

At the end of the process, $G^{*}$ will be transformed into an image including a set of feature points, which should represent the skeleton of the gray-tone image. Indeed, the set of feature points has not always a linear structure, so that it might not be correct to regard it as the skeleton of $G^{*}$. An example is shown in Fig. 2, concerning a part of magnified biological material scanned at 300 dpi, 256 gray-levels.

\subsection{Tricky Patterns}

The set of feature points we obtain by the previous process is not ensured to be onepixel-thick. Indeed, there are patterns in $G^{*}$, which are inhibited to be lowered because of the connectivity test, even if their lowering seems to be intuitively easy to achieve. 


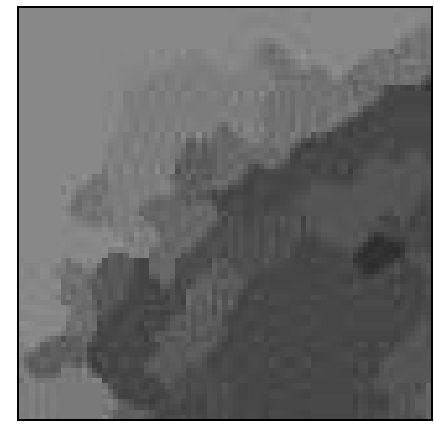

a)

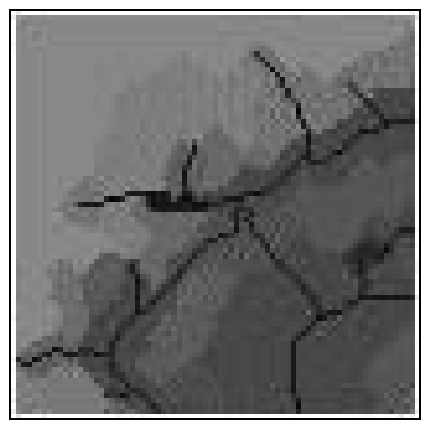

b)

Fig. 2. a) input image $G^{*}$. b) skeleton including a thick subset (superimposed over the input).

We don't mention here the simple cases of the type shown in Fig. 3, where the presence of pixels that are non simple points in the binary images $B_{k} \quad(k=6$ and $k=7)$ causes a local thickening of the skeleton. These patterns are also common when dealing with non gray-tone images [12], and their reduction to unit thickness is easy to obtain by using topology preserving operations in a postprocessing phase (namely, by lowering the underlined pixels 7).

$\begin{array}{lllllll}1 & 1 & 1 & 1 & 1 & 1 & 1 \\ 1 & 6 & 1 & 7 & 1 & 1 & 1 \\ 1 & 1 & 6 & \underline{7} & 1 & 1 & 1 \\ 1 & 1 & 1 & 6 & \underline{7} & 1 & 1 \\ 1 & 1 & 6 & 1 & 9 & 9 & 1 \\ 1 & 6 & 1 & 1 & 1 & 1 & 1 \\ 1 & 1 & 1 & 1 & 1 & 1 & 1\end{array}$

Fig. 3. Pixels with gray-value different from 1 are not allowed to change their status. Further lowering can be achieved during a postprocessing phase.

$\begin{array}{llllll}1 & 1 & 1 & 1 & 1 & 1 \\ 1 & 1 & \underline{6} & 1 & 1 & 1 \\ 1 & 1 & \underline{6} & 1 & 7 & 7 \\ 7 & 7 & 6 & 7 & 7 & 7 \\ 7 & 6 & 6 & 6 & 7 & 7 \\ 7 & 6 & 6 & 6 & 6 & 7 \\ 7 & 7 & 7 & 7 & 7 & 7\end{array}$

Fig. 4. Pixels with gray-value 6 cannot be lowered when topology and end points are preserved.

Here we refer to regions, even of considerable size, whose pixels are all detected as feature points. This occurs, for instance, for the regions with gray-value 6 in Fig. 4. Their pixels cannot be lowered because when the application of the topology preserving reduction operator starting from the lower border points reaches the underlined pixels, it turns out that the underlined pixels are either non simple points or end points, and all the successive more internal pixels, when checked, are non simple points because their connectivity number turns out to be equal to zero. 
Indeed, the problem arises as soon as an image subset is bordered almost completely by pixels with higher gray-values, and the subset can communicate with an adjacent region with lower gray-value only through non simple points belonging to a narrow one-pixel-wide path.

A variation of the previous pattern may be as in Fig. 5a, where the gray-values of the pixels are ordered as follows: $a<b<c<d$. In Fig. 5b, pixels $b$ are shown as labeled with their $(3,4)$-weighted distance from the region with lower gray-value including pixels $a$.

$\begin{array}{llllllllll}a & a & d & d & d & d & d & d & d & d \\ a & a & b & b & b & b & b & b & b & d \\ a & a & b & b & b & b & b & b & b & d \\ a & a & b & b & b & b & b & b & b & d \\ b & b & b & b & b & b & b & b & b & d \\ a & a & a & d & c & c & c & c & c & d \\ a & a & a & d & c & c & c & c & c & d \\ a & a & a & d & c & c & c & c & c & d \\ a & a & a & d & d & d & d & d & d & d\end{array}$

a)

$\begin{array}{llllllllll}a & a & d & d & d & d & d & d & d & d \\ a & a & 3 & 6 & 9 & 12 & 15 & 18 & 21 & d \\ a & a & 3 & 6 & 9 & 12 & 15 & 18 & 21 & d \\ a & a & 3 & 6 & 8 & 11 & 14 & 17 & 20 & d \\ \underline{3} & \underline{3} & \underline{3} & \underline{4} & \underline{7} & \underline{10} & \underline{13} & \underline{16} & \underline{19} & d \\ a & a & a & d & c & c & c & c & c & d \\ a & a & a & d & c & c & c & c & c & d \\ a & a & a & d & c & c & c & c & c & d \\ a & a & a & d & d & d & d & d & d & d\end{array}$

b)

Fig. 5. a) Four regions, where the gray-values of the pixels are $a<b<c<d$. b) The set of $c$ 's cannot be lowered, due to the presence of the underlined feature points.

It is straightforward to see that the underlined pixels are detected as feature points. In particular, these pixels result currently internal for all the distance labels starting from the underlined pixel labeled 4.

When the set of c's has to be processed, it happens that the lower border points cannot be lowered because they appear to be internal pixels (i.e., with connectivity number equal to 0 ) due to the presence of the non simple points detected on the set of $b$ 's. In turn, if lower border points are not lowered, also the remaining $c$ 's cannot be lowered because they are not adjacent to any pixel with lower gray-value. Thus, the set of $c$ 's is not modified.

To deal with the kind of patterns shown above in Figs. 3 and 4, our approach is to break the barrier (constituted by one or more non simple points) which prevents lowering of the pixels of an irreducible region.

To this purpose, once a pixel $p$ is found that is detected as non simple point because it appears to be internal and $p$ has at least one d-neighbor belonging to a region 
with higher gray-value, we temporarily interrupt the lowering process and check the number and position of such d-neighbors. If there is only one d-neighbor or a pair of two opposite d-neighbors, we induce lowering of $p$ and of all its neighbors with the same gray-value of $p$ not yet examined, and at the same time we mark as feature points the d-neighbors with higher gray-value. Then, the lowering process is resumed. This induced lowering is just a labeling operation which creates the conditions allowing the modification of the irreducible region. Marking of the d-neighbors having higher gray-value ensures the connectedness of the set of feature points (hence of the skeleton). What has to be paid for the success of this operation is that topology may not be preserved. For instance in Fig. 4 the pixels 6 not underlined are lowered to 1 , so that the binary image $B_{6}$ has initially the foreground constituted by one simply connected component, while after the process that component includes also one hole. On the other hand, in the case of Fig. 5 topology is preserved. Anyway, as far as skeletonization of gray-tone images is concerned, topology preservation is not a fundamental issue [13]. As for the example mentioned in Fig. 2, the onepixel-wide skeleton is shown in Fig. 6.

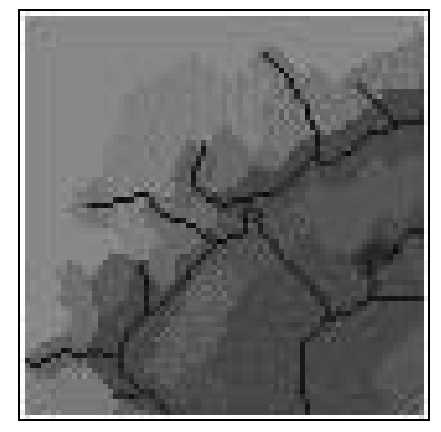

Fig. 6. One-pixel-wide modification of the skeleton shown in Fig. 2.

\subsection{Postprocessing}

Generally, the found gray-skeleton is not everywhere perceptually meaningful since it may include a number of branches which either are created in correspondence of end points which are not significant, or are found as lines dividing two bottoms at least one of which is not significantly deep. In this paper, we don't deal specifically with this phase and refer to the recent literature for a discussion regarding suitable criteria of significance $[13,14]$.

\section{Conclusion}

We have described a sequential algorithm for the skeletonization of a gray-tone image. We have regarded the image as constituted by a number of constant gray-value regions, and have looked for a set of digital lines that is mainly placed centrally in 
correspondence with regions with locally higher gray-values. This set has been detected on the Distance Transform of the image, computed according to the $(3,4)$ weighted distance by ordered propagation over regions with increasing gray-value. An advantage of using the Distance Transform is that it creates a structure in the interior of each region, and favors the detection of skeleton subsets in correspondence with elongated regions not strongly dominated by other regions.

Topology preserving reduction operations have been used to lower the gray-value of simple points. However, differently from the binary case, where the use of the connectivity test is generally sufficient to create a one-pixel-thick skeleton, we need to consider also a different operation including a suitable labeling of the neighborhood of the pixel under examination. In this way, we are able to obtain a one-pixelthick skeleton in correspondence with some of the tricky subsets of the gray-tone image that can be regarded as irreducible.

\section{References}

1. Wang, L., Pavlidis, T.: Detection of curved and straight segments from grey scale topography. CVGIP: Image Understanding 58 (1993) 352-365

2. Beucher, S., Meyer, F.: The morphological approach to segmentation: the watershed transformation. In: Dougherty, E.R. (ed.): Mathematical Morphology in Image Processing. Marcel Dekker, New York (1993) 433-481

3. Goetcherian, V.: From binary to grey tone image processing using fuzzy logic concepts. Pattern Recognition 12 (1980) 7-15

4. Bertrand, G., Everat, J.-Ch., Couprie, M.: Image segmentation through operators based on topology. J. Electronic Imaging 6 (1997) 395-405

5. Rosenfeld, A.: On connectivity properties of greyscale pictures. Pattern Recognition 16 (1983) 47-50

6. Piper, J., Granum, E.: Computing distance transformations in convex and non-convex domains. Pattern Recognition 20 (1987) 599-615

7. Borgefors, G.: Distance transformations in digital images. Computer Vision, Graphics and Image Processing 34 (1986) 344-371

8. Gilbert, E. N.: Lattice-theoretic properties of frontal switching functions. J. Math. Phys. 33 (1954) 57-67

9. Yokoi, S., Toriwaki, J.-I., Fukumura, T.: An analysis of topological properties of digitized binary pictures using local features. Computer Graphics and Picture Processing 4 (1975) 63-73

10. Arcelli, C.: Topological changes in grey-tone digital pictures. Pattern Recognition 32 (1999) 1019-1023

11. Wang, Y., Bhattacharya, P.: On parameter-dependent connected components of gray images. Pattern Recognition 29 (1996) 1359-1368

12. Arcelli, C., Sanniti di Baja, G.: Skeletons of planar patterns. In: Kong, T.Y., Rosenfeld, A. (eds): Topological Algorithms for Digital Image Processing. North Holland, Amsterdam (1996) 99-143

13. Arcelli, C., Serino, L.: Regularization of graphlike sets in gray-tone digital images. Int. J. Pattern Recognition and Artificial Intelligence 15 (2001) 643-657

14. Najman, L., Schmitt, M.: Geodesic saliency of watershed contours and hierarchical segmentation. IEEE Trans. on PAMI 18 (1996) 1163-1173 